\title{
Psychological interventions for post-traumatic stress disorder and depression in young survivors of mass violence in low- and middle-income countries: meta-analysis ${ }^{\dagger}$
}

\author{
Nexhmedin Morina, Mina Malek, Angela Nickerson and Richard A. Bryant
}

\section{Background}

The majority of survivors of mass violence live in low- and middle-income countries (LMICS).

\author{
Aims \\ To synthesise empirical findings for psychological \\ interventions for children and adolescents with post- \\ traumatic stress disorder (PTSD) and/or depression in LMICS \\ affected by mass violence.
}

\section{Method}

Randomised controlled trials with children and adolescents with symptoms of PTSD and/or depression in LMICs were identified. Overall, 21812 records were found through July 2016 in the Medline, PSycINFO and PILOTS databases; 21 met the inclusion criteria and were reviewed according to recommended guidelines.

\section{Results}

Twenty-one studies were included. Active treatments for
PTSD yielded large pre-treatment to post-treatment changes $(g=1.15)$ and a medium controlled effect size $(g=0.57)$. Effect sizes were similar at follow-up. Active treatments for depression produced small to medium effect sizes. Finally, after adjustment for publication bias, the imputed uncontrolled and controlled effect sizes for PTSD were medium and small respectively.

\section{Conclusions}

Psychological interventions may be effective in treating paediatric PTSD in LMICS. It appears that more targeted approaches are needed for depressive responses.

\section{Declaration of interest}

None.

\section{Copyright and usage}

(c) The Royal College of Psychiatrists 2017.
Approximately half of the world's countries have been affected by war or open conflict in the past three decades. ${ }^{1,2}$ Most war casualties are civilians, ${ }^{3}$ and the overwhelming majority of them live in low- and middle-income countries (LMICs). ${ }^{4}$ Post-traumatic stress disorder (PTSD) and depression are the most prevalent mental disorders in war-affected communities, and the prevalence rates of these conditions are significantly higher in such communities than in communities with no recent history of open conflict. ${ }^{5,6}$ This pattern applies to both adults and children, with more than $80 \%$ of the world's population of young people living in LMICs. PTSD is associated with mental and physical dysfunction, ${ }^{7}$ is chronic if untreated, ${ }^{8}$ and contributes significantly to the burden of disease. ${ }^{9}$ As a result, mitigating the effects of trauma and adversity has become a major global public health issue. ${ }^{10-12}$ The challenges for implementing evidence-based interventions in LMICs include insufficient mental health services, inadequately qualified practitioners to deliver interventions and impediments to adapting established interventions to the needs of LMIC contexts. ${ }^{13}$ Numerous controlled trials of psychological interventions for mental health problems in children and youths following mass violence have been conducted in LMICs. A meta-analysis by Tol et al in 2011 that included four randomised controlled trials (RCTs) in children and adolescents exposed to mass traumatic events produced small effects on PTSD when psychosocial interventions were compared with waiting list or usual care. ${ }^{14}$ These authors further concluded that the analysed trials had serious limitations in study design. Our aim was to survey the published literature on psychological interventions for children and young people in LMICs affected by mass violence

†See editorial, pp. 243-244, this issue. to provide an updated meta-analytic review of the effects of psychological interventions on PTSD and depression symptoms.

\section{Method}

We defined the main structured research question describing the Population, Intervention, Comparison, Outcome and Study (PICOS) design in accordance with the recommendations of the Preferred Reporting Items for Systematic Reviews and Metaanalyses (PRISMA) group. ${ }^{15}$ Our aim was to elucidate whether psychological treatment of children and adolescents improved war-related PTSD and/or depression symptoms compared with control conditions in RCTs.

\section{Identification and selection of studies}

Our inclusion criteria were that the study was conducted in an LMIC with survivors of mass violence who were 19 years old or younger, assignment to treatment conditions was random and at least 10 participants completed a psychological treatment aimed at reducing PTSD or depression (or both). No restriction was made as to intervention format, publication type, year of publication or publication language. A trial was excluded if the majority of participants were older than 19 years. If publications did not provide sufficient data for meta-analysis, the authors were contacted by email up to twice to retrieve these data. A trial was labelled as being conducted with survivors of mass violence if the conflict in question had taken place in a country that was listed as a war-affected country by the Uppsala Conflict Data Program. ${ }^{1,16}$ This database defines wars as mass conflicts that generate 1000 or more battle-related deaths in one calendar year. We searched the PsycINFO, Medline and PILOTS databases to 
9 July 2016. The following search terms were used in the fields 'title', 'abstract' and 'key concepts': posttraumatic stress OR PTSD OR depress* OR MDD AND genocide or war* OR prisoners of war OR mass violence OR mass conflict ${ }^{\star}$ OR post-conflict ${ }^{\star}$ OR political conflict ${ }^{\star}$ OR armed conflict* OR ethnic cleansing OR terrorism OR torture OR persecution OR civilian* AND treatment ${ }^{\star}$ OR intervention* OR therapy OR psychotherapy OR exposure OR trial. Additionally, reference lists of included publications and other relevant reviews were examined to retrieve additional studies.

Two authors (N.M. and M.M.) coded and extracted from each study the treatment target (PTSD or depression or both); number of participants; comparison group; type of outcome measure used (self-report measurement or structured interview); intervention format (individual or group); therapist (trained lay or professional therapist); type of intervention; number of sessions; country; and gender. If reported, scores of general function impairment were also coded and extracted. The pre-treatment, post-treatment and follow-up assessment mean and standard deviation scores and/ or mean and standard deviation change scores for each outcome measure also were recorded. If mean age or gender proportions were not reported separately for each condition, the reported average data were used for all conditions.

\section{Quality assessment}

The Cochrane Collaboration's tool for assessing risk of bias was used for evaluating the quality of the studies regarding selection bias (random sequence generation and allocation concealment), detection bias, attrition bias, reporting bias and other sources of bias. ${ }^{17}$ Risk of bias in each domain was classified as low, high or unclear. Two raters independently rated all studies.

\section{Coding of treatment characteristics}

Treatment interventions were first coded as either 'active treatment' or 'control group'. Next, active treatments were subdivided into trauma-focused cognitive-behavioural therapy (CBT), treatments mainly focusing on the memory of the trauma and/or its meaning; CBT without a main focus on the traumatic memory; multidisciplinary treatment (treatments including intervention techniques from different therapeutic approaches); classroom-based interventions for students; interpersonal psychotherapy; and meditation. Control conditions were subdivided into waiting-list or non-active controls and active control conditions. Active control conditions consisted of supportive counselling, creative play or treatment as usual (TAU). Finally, all active treatments were categorised according to whether they were delivered as individual or group treatment.

\section{Statistical analysis}

Intention-to-treat (ITT) samples were used when available (nine publications) and completer samples were used if ITT samples were not provided (nine publications). Given the limited number of controlled trials meeting our criteria, we found it informative first to compute within-group effect sizes for the impact of treatment on PTSD symptoms (i.e. change from pre-treatment to post-treatment and follow-up, respectively). However, as uncontrolled effect sizes do not account for the impact of time on symptoms, we viewed controlled effect sizes as more robust for assessing the efficacy of treatment effect. For uncontrolled effect sizes the post-treatment or follow-up mean was subtracted from the pre-treatment mean, and for controlled effect sizes the control group mean was subtracted from the treatment group mean at post-treatment or follow-up and divided by the pooled standard deviation. Subsequently, to obtain the effect size Hedges' $g$, the outcome was multiplied by a sample size correction factor
$J=1-(3 /(4$ d.f. -1$)){ }^{18}$ Subgroup analyses were conducted if a specific group of interventions consisted of at least four trials. As experimental conditions were mostly compared with waitinglist conditions, we separately analysed the overall effect of waiting-list control groups. Analyses were completed with Comprehensive Meta-Analysis version 3, using the random effects model to calculate effect sizes given the heterogeneity of the studies. ${ }^{19,20}$ Effect sizes may be conservatively interpreted with Cohen's convention of small (0.2), medium (0.5) and large (0.8) effects. $^{21}$ To test homogeneity of effect sizes we calculated the Q-statistic and the $I^{2}$ statistic which is an indicator of heterogeneity in percentages, with higher percentages indicating high heterogeneity. The risk of publication bias was assessed for the primary outcome measures through visual inspection of the funnel plot (for analyses including more than nine trials) and by examining the relation between effect and sample sizes, with relatively higher effect sizes of smaller studies being an indicator of publication bias. ${ }^{22}$ Furthermore, we calculated the likely number of missing studies using Duval \& Tweedie's trim and fill procedure, ${ }^{23}$ which yields an estimate of the effect size after publication bias has been taken into account.

\section{Results}

In total 21 studies fulfilled our criteria (Fig. 1, Table 1). Nineteen studies were published in peer-reviewed journals, ${ }^{24-42}$ one was a book, ${ }^{43}$ and one a doctoral thesis. ${ }^{44}$ All publications were in English. Nineteen publications reported on PTSD symptoms and 11 on depression symptoms. Most trials targeted both PTSD and depression $(k=10)$ and the remaining studies targeted either

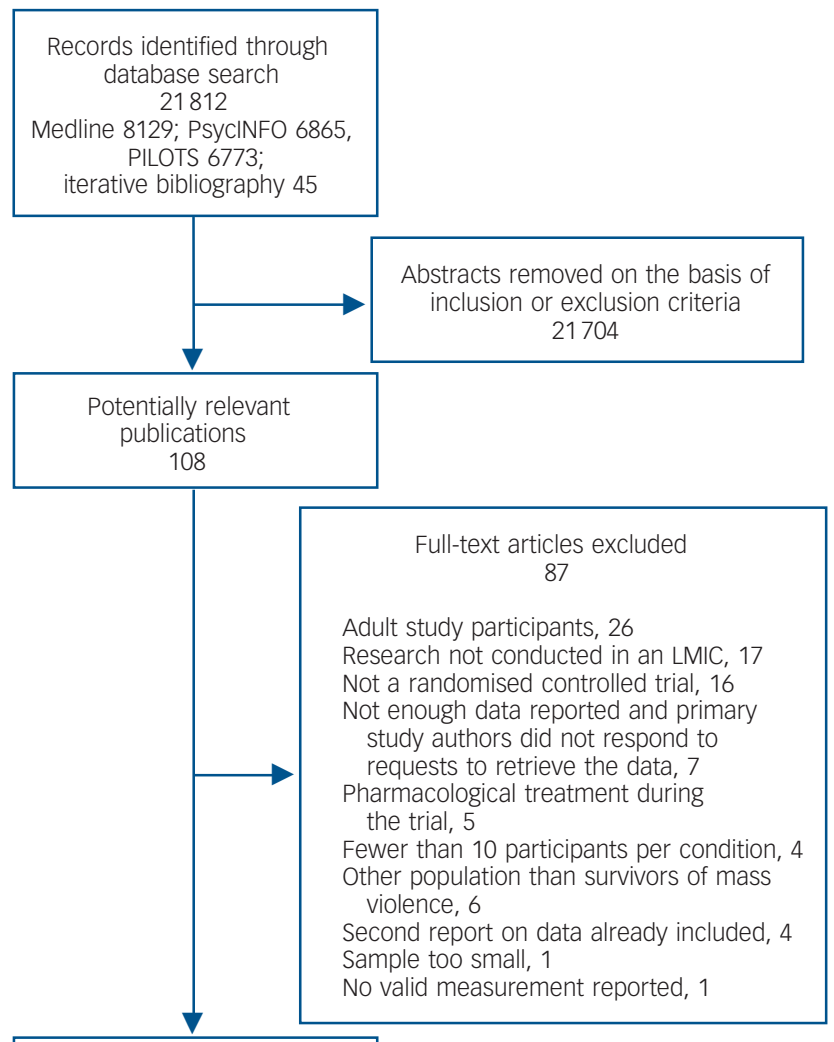

Trials included in meta-analysis 21

Fig. 1 Study selection process. LMIC, low- to middle-income country. 


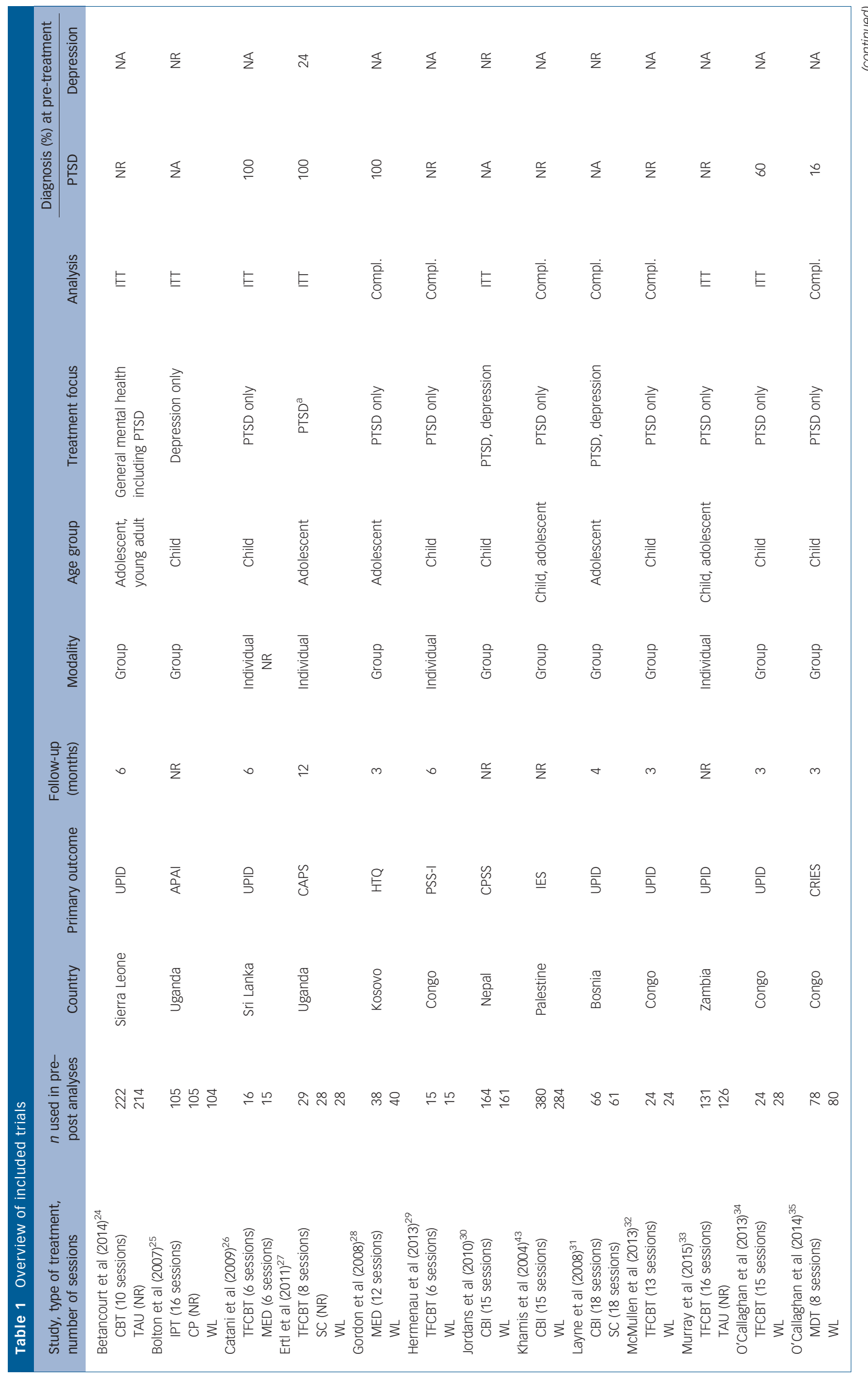




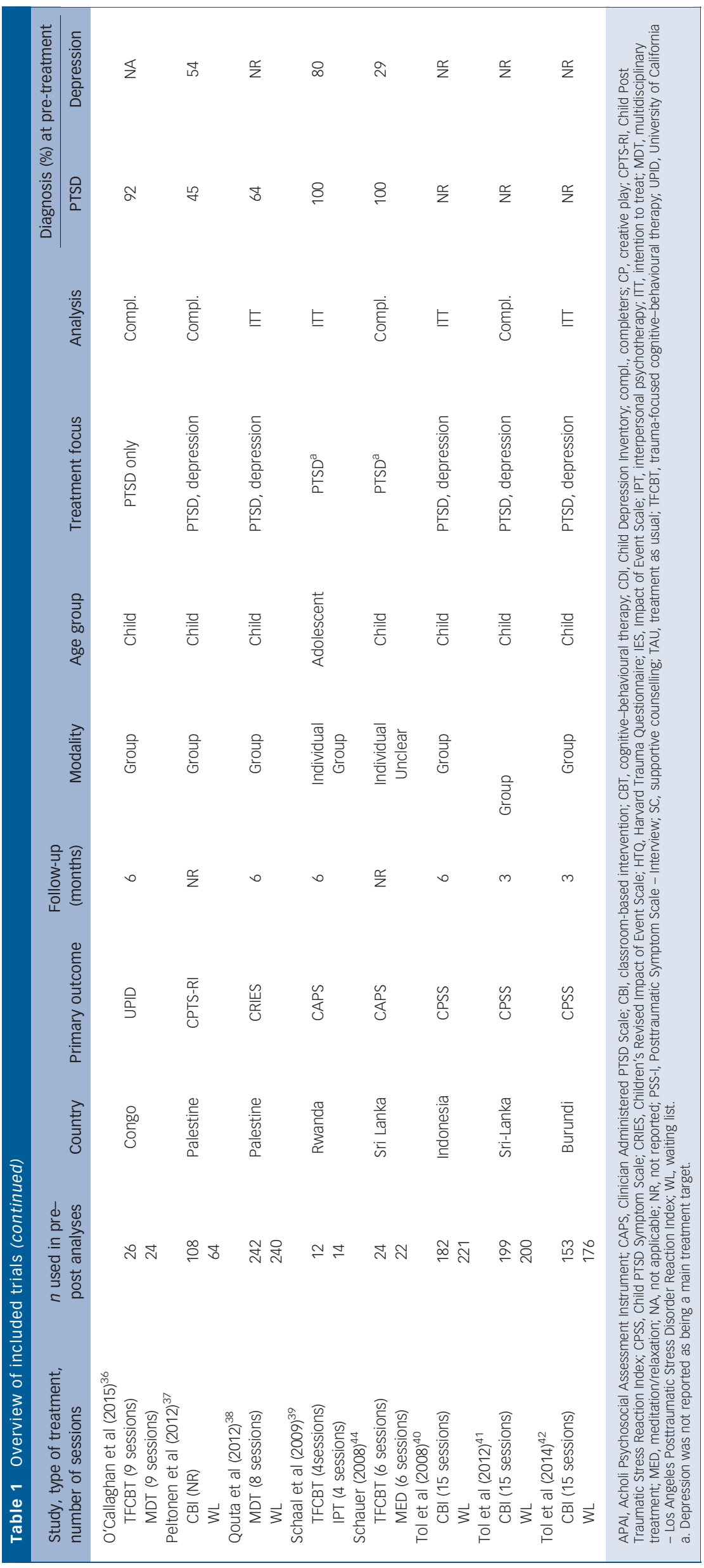


PTSD alone $(k=10)$ or depression $(k=1)$ alone. In total, 3444 treated participants and 1068 participants in a waiting-list condition were included in the analyses. In the 21 trials, 28 and 16 treatment arms targeted symptoms of PTSD and depression, respectively (Table 1). The difference between the number of trials and the number of interventions results from the fact that some trials assessed the efficacy of more than one active intervention. Active treatments were compared with waiting-list groups 13 and 8 times in relation to PTSD and depression symptoms, respectively. The number of participants per condition varied from 12 to 380 , with a mean of 102.5 (s.d.=91.0). The mean age of participants was 13.4 years $($ s.d. $=1.7)$ and half of the participants were female. Fifteen trials were conducted with children, four with adolescent participants and two with both groups combined. Of the trials focusing on both PTSD and depression, three reported treatment of PTSD as the major focus and additionally measured the efficacy of the intervention for depression among individuals with PTSD. ${ }^{27,39,44}$ The remaining seven had a general focus on treating war-related psychopathologic symptoms and focused on both PTSD and depression.

Both structured clinical interviews and self-report scales were used as primary outcome measures. All primary outcome measures provided continuous data. With regard to the 20 trials assessing PTSD, structured clinical interviews were used in four trials and self-report scales in the remaining 16 trials. However, self-report measures were mostly administered as interviews rather than completed by the participant. For the assessment of depression, structural clinical interviews were used in four trials, self-report measures administered as interviews in five trials, and self-reports filled out by the participant in two trials.

Ten studies reported the number of participants fulfilling diagnostic criteria for PTSD at the pre-treatment assessment and four studies reported those with depression. With regard to PTSD, five trials reported that all participants had PTSD at pre-treatment assessment and five studies reported prevalence rates of $45-92 \%$ (see Table 1). For depression the reported prevalence among the four studies in question ranged between $21 \%$ and $80 \%$. The number of treatment sessions implemented ranged from 4 to 18 (median 11.5, s.d. $=4.3$ ). Trauma-focused CBT interventions were associated with a lower mean number of treatment sessions. In fact, all interventions with six or fewer sessions were traumafocused CBT interventions conducted by the same group. Accordingly, the number of treatment sessions could not be used in subanalyses. Similarly, all but one of the trials of therapy conducted on an individual basis were conducted by the same group, and thus we refrained from comparing individual treatments with group treatments. The interventions were mainly conducted by non-psychotherapists who were trained for the purpose of the study, with only three trials making use of professionals to conduct the treatment. ${ }^{29,38,39}$ Interventions were mostly conducted in groups, with only six trials being implemented on an individual basis. Follow-up duration ranged from 3 months to 12 months. Given the limited number of included trials, all follow-up assessments regardless of the time of assessment were entered as one group in the meta-analysis.

\section{Effects on PTSD}

Uncontrolled effect sizes

Across all active interventions $(k=22)$ a large aggregated pre-post effect size was found: $g=1.15,95 \%$ CI $0.82-1.48$. With regard to uncontrolled effect sizes relating to the change from pre-treatment to follow-up, results showed large effect sizes across active treatments: $g=1.28,95 \%$ CI $0.98-1.57, k=16$ (Table 2).

\section{Controlled effect sizes}

The aggregated between-group effect size comparing active treatments $v$. control conditions at post-treatment $(k=16)$ was medium: $g=0.57,95 \%$ CI $0.27-0.88$. The comparison between active conditions with waiting-list only $(k=12)$ produced a medium effect size: $g=0.53,95 \%$ CI $0.25-0.81$ (Fig. 2, Table 3). The aggregated between-group effect size comparing active treatments with control conditions at follow-up was small: $g=0.23,95 \%$ CI $0.05-0.41 \quad(k=9)$. Four trials compared traumafocused CBT with other active conditions at post-treatment (interpersonal psychotherapy, multidisciplinary treatment and meditation), ${ }^{27,29,31,39}$ and results showed no difference between these interventions: $g=0.05,95 \%$ CI -0.26 to 0.36 . At follow-up only three studies compared trauma-focused CBT with other active conditions.

\section{Effects on depression}

\section{Uncontrolled effect sizes}

Across all interventions $(k=12)$ a small pre-post effect size was found: $g=0.30,95 \%$ CI $0.08-0.53$. Uncontrolled effect sizes for changes in depression symptoms from pre-treatment to follow-up showed a medium effect size: $g=0.52,95 \%$ CI $0.22-0.82(k=8)$.

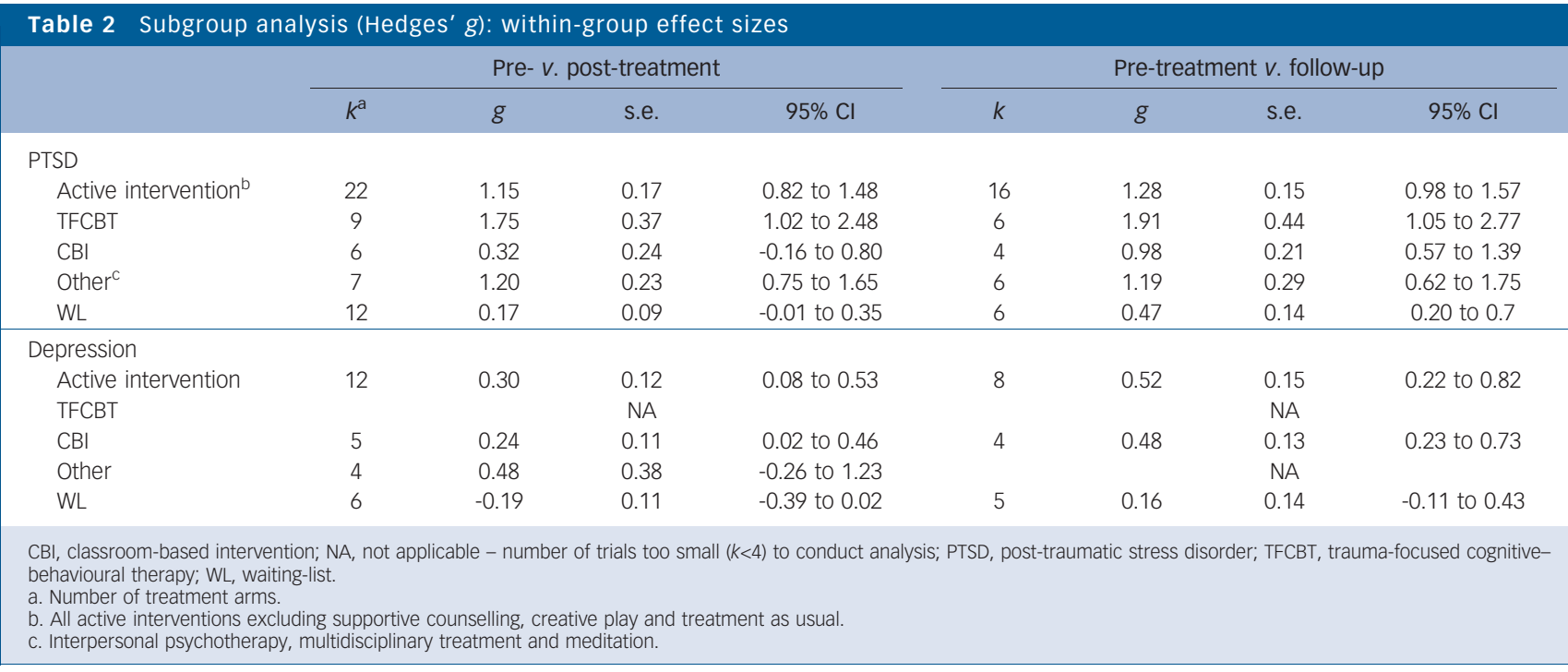




\begin{tabular}{|c|c|c|c|c|c|c|c|c|}
\hline & \multicolumn{4}{|c|}{ Post-treatment } & \multicolumn{4}{|c|}{ Follow-up } \\
\hline & $k^{a}$ & $g$ & s.e. & $95 \% \mathrm{Cl}$ & $k$ & $g$ & s.e. & $95 \% \mathrm{Cl}$ \\
\hline \multicolumn{9}{|l|}{ PTSD } \\
\hline Active $v \cdot W^{b}$ & 12 & 0.53 & 0.14 & 0.25 to 0.81 & \multirow[t]{4}{*}{6} & \multirow[t]{4}{*}{0.27} & 0.13 & \multirow[t]{4}{*}{0.02 to 0.52} \\
\hline TFCBT v. WL & 4 & 1.33 & 0.58 & 0.20 to 2.46 & & & NA & \\
\hline CBI v. WL & 5 & 0.14 & 0.15 & -0.16 to 0.44 & & & NA & \\
\hline Other $v . W L^{c}$ & & & NA & & & & NA & \\
\hline \multicolumn{9}{|l|}{ Depression } \\
\hline Active $v$. WL & 7 & 0.25 & 0.10 & 0.06 to 0.45 & 5 & \multirow[t]{4}{*}{-0.02} & 0.06 & \multirow[t]{4}{*}{-0.14 to 0.10} \\
\hline TFCBT $v$. WL & & & NA & & & & NA & \\
\hline $\mathrm{CBI}$ v. WL & 4 & 0.31 & 0.09 & 0.14 to 0.48 & & & NA & \\
\hline Other $v$. WL & \multicolumn{4}{|c|}{ NA } & \multicolumn{2}{|c|}{ NA } & & \\
\hline $\begin{array}{l}\text { CBI, classroom-base } \\
\text { cognitive-behavioura } \\
\text { a. Number of treatm } \\
\text { b. 'Active' comprise } \\
\text { c. 'Other' comprises }\end{array}$ & $\begin{array}{l}\text { JA, nc } \\
\text { vaitin } \\
\text { Jentio }\end{array}$ & $\begin{array}{l}\text { - num } \\
\text { supp } \\
\text { Itidisci }\end{array}$ & $\begin{array}{l}\text { als too } \\
\text { nselling } \\
\text { atment }\end{array}$ & $\begin{array}{l}<4) \text { to conduct an } \\
\text { ve play and treatme } \\
\text { editation. }\end{array}$ & $\begin{array}{l}\text { SD, } \mathrm{p} \\
\text { ual. }\end{array}$ & atic stress & r; TFCB & \\
\hline
\end{tabular}

\section{Controlled effect sizes}

The aggregated between-group effect size comparing active treatments with control conditions at post-treatment $(k=10)$ was small: $g=0.26,95 \%$ CI $0.07-0.45$. The comparison between active conditions with waiting-list only at post-treatment $(k=7)$ produced a small effect size: $g=0.25,95 \%$ CI $0.06-0.45$ (Fig. 2). At follow-up there was no difference between active treatments and control conditions: $g=-0.02,95 \% \mathrm{CI}-0.11$ to $0.08(k=7)$.

\section{Heterogeneity}

Heterogeneity was large for within-group effect sizes (PTSD: $I^{2}=96.8, Q=658.8$, d.f. $=21, \quad P<0.001$; depression: $I^{2}=91.0$, $Q=122.9$, d.f. $=11, P<0.001)$ as well as for between-group effect sizes (PTSD: $I^{2}=94.7, Q=284.5$, d.f. $=15, P<0.001$; depression: $I^{2}=79.8, Q=44.6$, d.f. $\left.=9, \quad P<0.001\right)$, indicating substantial heterogeneity in effect sizes between studies. Table 2 shows the aggregated effect sizes for the different types of treatment included. However, given the relatively small number of studies in most categories, the power was judged to be inadequate for direct comparison of the different groups of treatments.

\section{Effect on associated symptoms}

Four publications reported the effect of treatment on functional impairment and the aggregated pre-post effect size in these trials was medium: $g=0.65,95 \%$ CI $0.50-0.80$. When active conditions were compared with waiting-list controls post-treatment, the aggregated effect size was small to medium: $g=0.36,95 \%$ CI 0.26-0.49 $(k=4)$. At follow-up there was a small effect of active treatments compared with waiting-list controls: $g=0.18,95 \% \mathrm{CI}$ $0.06-0.30(k=4)$.

\section{Publication bias}

Inspection of the funnel plots depicting the uncontrolled effect sizes (pre- $v$. post-treatment) for PTSD and depression suggested the presence of asymmetry, as the direction of the effect of the smaller trials was towards the right. This indicates that nonsignificant trials might not have been published. A similar pattern applied to PTSD trials comparing active treatments with waitinglist conditions at post-treatment (as fewer than ten trials compared active treatments for depression with waiting-list

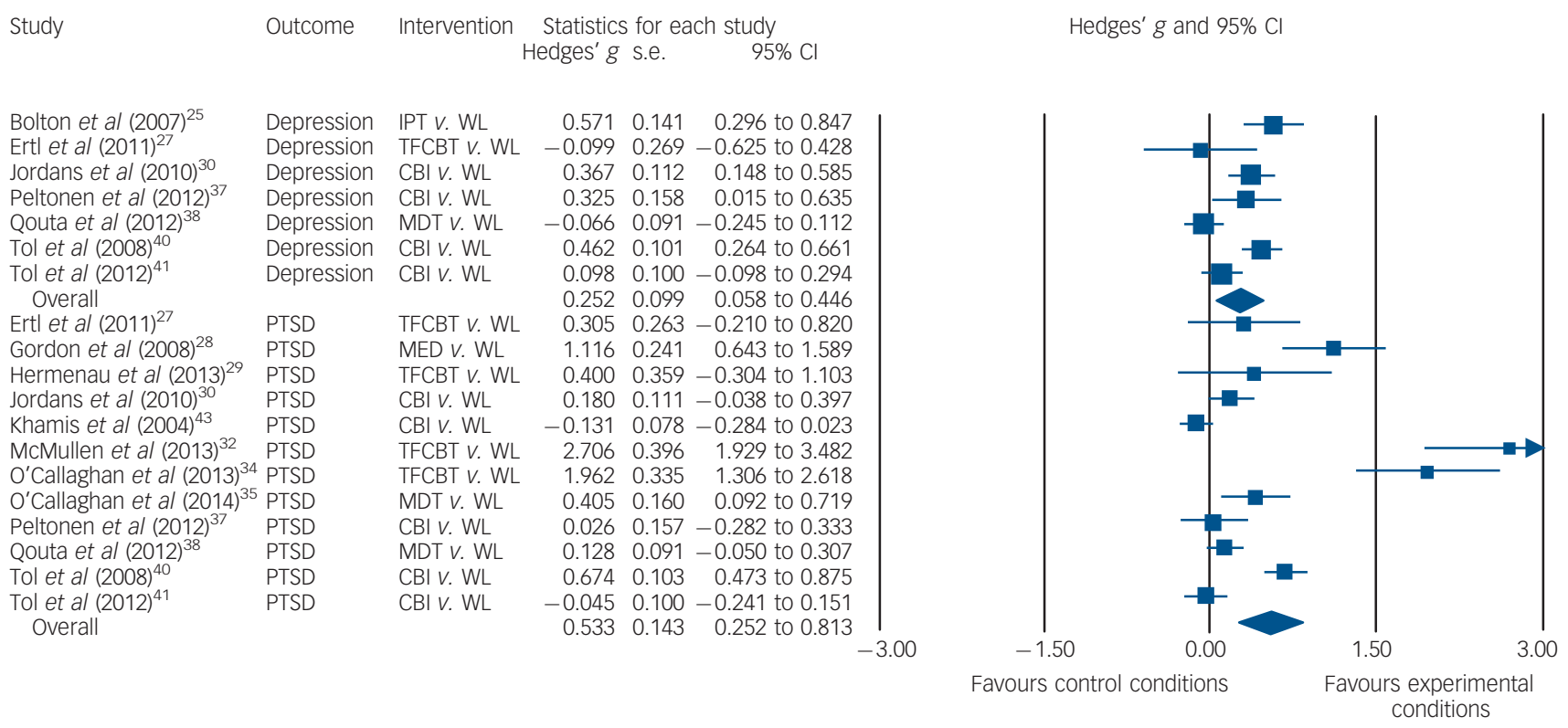

Fig. 2 Forest plot of controlled effect sizes comparing active treatments with waiting-list controls at post-treatment assessment. $\mathrm{CBI}$, classroom-based intervention; IPT, interpersonal psychotherapy; MDT, multidisciplinary treatment; MED, meditation; PTSD, post-traumatic stress disorder; TFCBT, trauma-focused cognitive-behavioural therapy; WL, waiting list. 
controls, publication bias could not be assessed in this regard). ${ }^{22}$ The trim and fill procedure identified ten, two and seven studies to be missing regarding the uncontrolled effect sizes for PTSD and depression, and controlled effect size for PTSD, respectively. The produced imputed point estimates resulting from these three analyses were $0.34,0.11$ and 0.04 , respectively.

\section{Risk of bias in included trials}

The intraclass correlation coefficient of the total score for all studies combined as rated by the two raters was 0.88 (95\% CI 0.84-0.92), indicating good interrater reliability. The majority of the trials were rated as having low risk of bias in the domains random sequence generation (16 trials) and other bias (18 trials). The numbers of trials rated as having high or unknown risk in the specific domains were as follows: allocation concealment 10 trials, detection bias 11 trials, attrition bias 11 trials, reporting bias 17 trials. Altogether, the risk of bias was rated as low in $71 \%$ of cases. Furthermore, the trials did not differ substantially on risk of bias and thus this variable could not be included in subanalyses.

\section{Discussion}

Current published research indicates that psychological treatments may effectively treat PTSD in children and adolescents in LMICs. However, significant publication bias was found, and after adjustment for this bias the imputed uncontrolled and controlled effect sizes for PTSD were medium and small, respectively. Furthermore, the effects on depression were small, and here too publication bias was found. It is proposed that mass violence does not simply result in psychopathologic disorder emanating from the impact of war-related trauma but that mental health is also strongly affected by the subsequent social upheaval and other ongoing stressors. ${ }^{45,46}$ Nevertheless, our findings indicated that treatments focusing on memories of the traumatic experience produced the largest effects on PTSD. However, this result is limited by the low number of trials and potential publication bias. Of the four trials comparing trauma-focused CBT with a waitinglist group, two reported very large effect sizes and two small effect sizes, which may indicate publication bias.

The low effect sizes for depression may be attributed to several factors. First, not all studies included depression measures and so the limited variance may have skewed this finding. Second, depression may be more resistant to change in LMICs because of the ongoing adversity that people often have to manage, which may engender feelings of helplessness and hopelessness. Third, a substantial proportion of the interventions were designed to address PTSD rather than depression, and so it may not be surprising that depression reduced to a lesser extent. In fact, the two studies that employed interpersonal psychotherapy - a treatment with good evidence as a psychotherapy for depression - showed a strong effect for depression. ${ }^{25,39}$ It should also be noted that patients allocated to waiting-list conditions enjoyed a reasonable effect size in reduction of PTSD symptoms at follow-up (0.47), a result also found in treatments in high-income countries. $^{47}$

\section{Limitations}

We note some limitations to the treatments. First, approximately half the trials reported treatment completer data and the remainder reported ITT analyses; this heterogeneity in handling missing values raised difficulties in interpretation of results for a metaanalysis. Second, the variability in assessment by interview and self-report potentially leads to more heterogeneity in response as a result of measurement artefact. There were also limitations to our meta-analysis. The actual number of studies reviewed was small, making it difficult to conduct subanalyses of different groups. We also note the range of measures employed in different countries, which raises the potential problem of the lack of standardisation and suitability of measures across cultural contexts. ${ }^{48}$ Finally, we recognise that the focus on PTSD and depression ignored other important constructs in post-conflict settings, such as functioning.

\section{Study implications}

There is a need for caution about the capacity of these interventions to be implemented in LMICs at population levels. Publication bias might have led to an overestimation of the efficacy of interventions for mental health needs of young survivors of war. Furthermore, the mean number of treatment sessions prescribed by the treatment protocols was more than ten. This can be a significant barrier in LMICs because of difficulties for parents in transporting children to the site of intervention, civil conflict disrupting treatment access or priority being given to more immediate needs (e.g. working to assist family income). Further, many of the studies required considerable training of the providers of the treatment, as well as sustained supervision, to ensure that the intervention was delivered adequately. ${ }^{25}$ A central tenet of global mental health is that an intervention needs to be both efficacious and capable of being scaled up to the point that it can be readily implemented in LMICs despite the limited resources of these countries. ${ }^{49}$ A major challenge for global mental health is to develop more low-intensity interventions that can still achieve reasonable effect sizes but simultaneously provide cost-effective solutions to LMICs. This would enhance the long-term and sustainable capacity to disseminate the interventions to large numbers of children in need.

\footnotetext{
Nexhmedin Morina, PhD, Department of Psychology, University of Münster, Münster, Germany; Mina Malek, MS, Department of Psychology, University of Amsterdam, Amsterdam, The Netherlands; Angela Nickerson, PhD, Richard A. Bryant, PhD, School of Psychology, University of New South Wales, NSW, Sydney, Australia

Correspondence: Nexhmedin Morina, University of Münster, Fliednerstr. 21, 48149 Münster, Germany. Email: morina@uni-muenster.de

First received 16 Dec 2015, final revision 13 Oct 2016, accepted 16 Oct 2016
}

\section{Acknowledgements}

This work was supported by a grant from the Netherlands Institute for Advanced Study (NIAS) given to N.M. The NIAS had no role in any aspect pertinent to the meta-analysis or the writing of the manuscript or the decision to submit it for publication. The corresponding author (N.M.) had full access to all the data and had final responsibility for the decision to submit for publication.

\section{References}

1 Pettersson T, Wallensteen P. Armed conflicts, 1946-2014. J Peace Res 2015; 52: $536-50$.

2 Marshall MG, Cole BR. Global Report 2009: Conflict, Governance, and State Fragility. George Mason University Center for Systemic Peace and Center for Global Policy, 2009.

3 Bartov O. Mirrors of Destruction: War, Genocide and Modern Identity. Oxford University Press, 2000

4 Brundtland $\mathrm{GH}$. Mental health of refugees, internally displaced persons and other populations affected by conflict. Acta Psychiatr Scand 2000; 102: 159-61. 5 Steel Z, Chey T, Silove D, Marnane C, Bryant RA, van Ommeren M. Association of torture and other potentially traumatic events with mental health outcomes among populations exposed to mass conflict and displacement: a systematic review and meta-analysis. \#JAMA\# 2009; \#302\#: 537-49. 
6 Priebe S, Bogic M, Ajdukovic D, Franciskovic T, Galeazzi GM, Kucukalic A, et al. Mental disorders following war in the Balkans: a study in 5 countries. Arch Gen Psychiatry 2010; 67: 518-28.

7 Nemeroff CB, Bremner JD, Foa EB, Mayberg HS, North CS, Stein MB. Posttraumatic stress disorder: a state-of-the-science review. J Psychiatr Res 2006; 40: 1-21.

8 Morina N, Wicherts JM, Lobbrecht J, Priebe S. Remission from post-traumatic stress disorder in adults: a systematic review and meta-analysis of long term outcome studies. Clin Psychol Rev 2014; 34: 249-55.

9 Kessler RC, Aguilar-Gaxiola S, Alonso J, Chatterji S, Lee S, Ormel J, et al. The global burden of mental disorders: an update from the WHO World Mental Health (WMH) surveys. Epidemiol Psichiatr Soc 2009; 18: 23-33.

10 Collins PY, Patel V, Joestl SS, March D, Insel TR, Daar AS, et al. Grand challenges in global mental health. Nature 2011; 475: 27-30.

11 World Health Organization. Caring for Children and Adolescents with Mental Disorders. WHO, 2003.

12 Belfer ML, Remschmidt $\mathrm{H}$, Nurcombe B, Okasha A, Sartorius N. A global programme for child and adolescent mental health: a challenge in the new millenium. In The Mental Health of Children and Adolescents: An Area of Global Neglect (eds H Remschmidt, B Nurcombe, ML Belfer, N Sartorius, A Okasha): 11-111. Wiley, 2007.

13 Morina N, Rushiti F, Salihu M, Ford JD. Psychopathology and well-being in civilian survivors of war seeking treatment: a follow-up study. Clin Psychol Psychother 2010; 17: 79-86.

14 Tol WA, Barbui C, Galappatti A, Silove D, Betancourt TS, Souza R, et al. Mental health and psychosocial support in humanitarian settings: linking practice and research. Lancet 2011; 378: 1581-91.

15 Moher D, Liberati A, Tetzlaff J, Altman DG, PRISMA Group. Preferred reporting items for systematic reviews and meta-analyses: the PRISMA statement. J Clin Epidemiol 2009; 62: 1006-12.

16 Uppsala Conflict Data Program. UCDP Conflict Encyclopedia. UCDP (www.ucdp.uu.se/database).

17 Higgins JPT, Altman DG, Sterne JAC. Assessing risk of bias in included studies. In Cochrane Handbook for Systematic Reviews of Interventions Version 5.1.0 (eds JPT Higgins, S Green). Cochrane Collaboration, 2011.

18 Lipsey MW, Wilson DB. Practical Meta-Analysis. Sage, 2001.

19 Borenstein M, Hedges LV, Higgins JPT, Rothstein HR. Software. In Introduction To Meta-Analysis. Wiley, 2009.

20 Field AP, Gillett R. How to do a meta-analysis. Br J Math Stat Psychol 2010 63: 665-94.

21 Cohen J. Statistical Power Analysis for the Behavioral Sciences. Erlbaum, 1988.

22 Sterne JAC, Sutton AJ, Ioannidis JPA, Terrin N, Jones DR, Lau J, et al. Recommendations for examining and interpreting funnel plot asymmetry in meta-analyses of randomised controlled trials. BMJ 2011; 343: d4002.

23 Duval S, Tweedie R. Trim and fill: a simple funnel-plot-based method of testing and adjusting for publication bias in meta-analysis. Biometrics 2000; 56: 455-63.

24 Betancourt TS, McBain R, Newnham EA, Akinsulure-Smith AM, Brennan RT, Weisz JR, et al. A behavioral intervention for war-affected youth in Sierra Leone: a randomized controlled trial. J Am Acad Child Adolesc Psychiatry 2014; 53: 1288-97.

25 Bolton P, Bass J, Betancourt T, Speelman L, Onyango G, Clougherty KF, et al. Interventions for depression symptoms among adolescent survivors of war and displacement in northern Uganda: a randomized controlled trial. JAMA 2007; 298: 519-27.

26 Catani C, Kohiladevy M, Ruf M, Schauer E, Elbert T, Neuner F. Treating children traumatized by war and tsunami: a comparison between exposure therapy and meditation-relaxation in north-east Sri Lanka. BMC Psychiatry 2009; 9: 22

27 Ertl V, Pfeiffer A, Schauer E, Elbert T, Neuner F. Community-implemented trauma therapy for former child soldiers in northern Uganda: a randomized controlled trial. JAMA 2011; 306: 503-12.

28 Gordon JS, Staples JK, Blyta A, Bytyqi M, Wilson AT. Treatment of posttraumatic stress disorder in postwar Kosovar adolescents using mind-body skills groups: a randomized controlled trial. J Clin Psychiatry 2008; 69: 1469-76.

29 Hermenau K, Hecker T, Schaal S, Maedl A, Elbert T. Addressing posttraumatic stress and aggression by means of narrative exposure: a randomized controlled trial with ex-combatants in the eastern DRC. J Aggression Maltreat Trauma 2013; 22: 916-34.

30 Jordans MJD, Komproe IH, Tol WA, Kohrt BA, Luitel NP, Macy RD, et al. Evaluation of a classroom-based psychosocial intervention in conflictaffected Nepal: a cluster randomized controlled trial. J Child Psychol Psychiatry 2010; 51: 818-26.

31 Layne CM, Saltzman WR, Poppleton L, Burlingame GM, Pasalic A, Durakovic E, et al. Effectiveness of a school-based group psychotherapy program for war-exposed adolescents: a randomized controlled trial. J Am Acad Child Adolesc Psychiatry 2008; 47: 1048-62.

32 McMullen J, O'Callaghan $P$, Shannon C, Black A, Eakin J. Group traumafocused cognitive-behavioural therapy with former child soldiers and other war-affected boys in the DR Congo: a randomised controlled trial. J Child Psychol Psychiatry 2013; 54: 1231-41.

33 Murray LK, Skavenski S, Kane JC, Mayeya J, Dorsey S, Cohen JA, et al. Effectiveness of trauma-focused cognitive behavioral therapy among traumaaffected children in Lusaka, Zambia: a randomized clinical trial. JAMA Pediatr 2015; 169: 761-9.

34 O'Callaghan P, McMullen J, Shannon C, Rafferty H, Black A. A randomized controlled trial of trauma-focused cognitive behavioral therapy for sexually exploited, war-affected Congolese girls. J Am Acad Child Adolesc Psychiatry 2013; 52: 359-69.

35 O'Callaghan P, Branham L, Shannon C, Betancourt TS, Dempster M, McMullen J. A pilot study of a family focused, psychosocial intervention with war-exposed youth at risk of attack and abduction in North-Eastern Democratic Republic of Congo. Child Abuse Neg/ 2014; 38: 1197-207.

36 O'Callaghan $\mathrm{P}$, McMullen J, Shannon C, Rafferty H. Comparing a trauma focused and non trauma focused intervention with war affected Congolese youth: a preliminary randomised trial. Intervention 2015; 13: 28-44.

37 Peltonen K, Qouta S, El Sarraj E, Punamaki R. Effectiveness of school-based intervention in enhancing mental health and social functioning among waraffected children. Traumatology 2012; 18: 37-46.

38 Qouta SR, Palosaari E, Diab M, Punamaki R. Intervention effectiveness among war-affected children: a cluster randomized controlled trial on improving mental health. J Trauma Stress 2012; 25: 288-98.

39 Schaal S, Elbert T, Neuner F. Narrative exposure therapy versus interpersonal psychotherapy. A pilot randomized controlled trial with Rwandan genocide orphans. Psychother Psychosom 2009; 78: 298-306.

40 Tol WA, Komproe IH, Susanty D, Jordans MJD, Macy RD, De Jong JTVM. School-based mental health intervention for children affected by politica violence in Indonesia - a cluster randomized trial. JAMA 2008; 300: 655-62.

41 Tol WA, Komproe IH, Jordans MJD, Vallipuram A, Sipsma H, Sivayokan S, et al. Outcomes and moderators of a preventive school-based mental health intervention for children affected by war in Sri Lanka: a cluster randomized trial. World Psychiatry 2012; 11: 114-22.

42 Tol WA, Komproe $\mathrm{IH}$, Jordans MJD, Ndayisaba A, Ntamutumba P, Sipsma H, et al. School-based mental health intervention for children in war-affected Burundi: a cluster randomized trial. BMC Med 2014; 12: 56.

43 Khamis V, Macy R, Coignez V. The Impact of the Classroom/Community/ Camp-Based Intervention (CBI) Program on Palestinian Children. Save the Children, 2004.

44 Schauer M. Trauma Treatment for Children in War: Build-up of an EvidenceBased Large-scale Mental Health Intervention in North-Eastern Sri Lanka [Doctoral thesis]. University of Konstanz, 2008.

45 Miller KE, Rasmussen A. War exposure, daily stressors, and mental health in conflict and post-conflict settings: bridging the divide between traumafocused and psychosocial frameworks. Soc Sci Med 2010; 70: 7-16.

46 De Jong JT, Komproe IH, Van Ommeren M, El Masri M, Araya M, Khaled N, et al. Lifetime events and posttraumatic stress disorder in 4 postconflict settings. JAMA 2001; 286: 555-62.

47 Hesser $\mathrm{H}$, Weise $\mathrm{C}$, Rief W, Andersson $\mathrm{G}$. The effect of waiting: a metaanalysis of wait-list control groups in trials for tinnitus distress. J Psychosom Res 2011; 70: 378-84.

48 Kohrt BA, Rasmussen A, Kaiser BN, Haroz EE, Maharjan SM, Mutamba BB, et al. Cultural concepts of distress and psychiatric disorders: literature review and research recommendations for global mental health epidemiology. Int J Epidemiol 2014; 43: 365-406.

49 Tol WA, Patel V, Tomlinson M, Baingana F, Galappatti A, Panter-Brick C, et al. Research priorities for mental health and psychosocial support in humanitarian settings. PLOS Med 2011; 8: e1001096. 